Supporting Information

\title{
Fragment-Based Computational Method for Designing GPCR
}

\section{Ligands}

Yan Li, ${ }^{1,3 *}$ Yaping Sun, ${ }^{2}$ Yunpeng Song, ${ }^{2}$ Dongcheng Dai, ${ }^{2}$ Zhixiong Zhao, ${ }^{1 \S}$ Qing Zhang, ${ }^{2 \ddagger}$ Wenge Zhong, ${ }^{2 \dagger}$ Liaoyuan A. Hu, ${ }^{2}$ Yingli Ma, ${ }^{2}$ Xun $\mathrm{Li}^{2},{ }^{*}$ and Renxiao Wang ${ }^{1,3,4^{*}}$

${ }^{1}$ State Key Laboratory of Bioorganic and Natural Products Chemistry, Center for Excellence in Molecular Synthesis, Shanghai Institute of Organic Chemistry, Chinese Academy of Sciences, 345 Lingling Road, Shanghai 200032, People's Republic of China

${ }^{2}$ Amgen Asia R\&D Center, Amgen Biopharmaceutical R\&D (Shanghai) Co., Ltd., Shanghai 201210, People's Republic of China

${ }^{3}$ Department of Medicinal Chemistry, School of Pharmacy, Fudan University, 826 Zhangheng Road, Shanghai 201203, People's Republic of China

${ }^{4}$ Shanxi Key Laboratory of Innovative Drugs for the Treatment of Serious Diseases Basing on Chronic Inflammation, College of Traditional Chinese Medicines, Shanxi University of Chinese Medicine, Taiyuan, Shanxi 030619, People's Republic of China

${ }^{\S}$ Current address: School of Stomatology and Medicine, Foshan University, Foshan, Guangdong 528000, People's Republic of China

Current address: Pharma Research and Early Development (pRED), Roche Innovation Center Shanghai, Building 5, 720 CaiLun Road, Shanghai 201203, People's Republic of China

${ }^{\dagger}$ Current address: Regor Therapeutics, Inc., Building No. 2, Chuangxiang Yuan, 576 Libing Road, Shanghai 201210, People's Republic of China

* Contact E-mail: kathyli@mail.sioc.ac.cn (Li, Y.); wangrx@mail.sioc.ac.cn (Wang, R.);xli02@amgen.com (Li, X.) 


\section{TABLE OF CONTENTS}

PART 1. Computational details in CIP-based ligand design ..................... 3

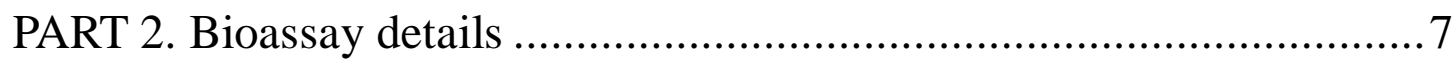

PART 3. MD simulations and MM-GB/SA calculation ........................... 9

PART 4. Summary of the candidate compounds for biological test ........13 


\section{PART 1. Computational details in CIP-based ligand design}

\section{GPCR-ligand interaction pattern analysis}

Similar to the interaction pattern at protein-protein binding interface proposed in our previous research, GPCR-ligand interaction pattern is defined as a fragment and three nearest residues around it. The similarity calculations for two pairs of interaction patterns include the fragment similarity and the spatial similarity. The fragment similarity is calculated based on the Tanimoto similarity described in the main text. The spatial similarity is calculated as the root-mean-square deviation (RMSD) value of two interaction patterns. In this process, the first thing is to check whether the residues are matched. According to the physicochemical similarity of their side chain, 20 natural amino acid residues are categorized into 9 groups: (1) residues with a small side chain: $\mathrm{G}$ and $\mathrm{A}$; (2) residues with a bulky hydrophobic side chain: V, L, I, and M (3) residues with an aromatic side chain: F, Y and W; (4) residues with a side chain that has a hydroxyl or thiol group: $\mathrm{S}, \mathrm{T}$ and $\mathrm{C}$; (5) residues with a side chain that has a carboxylic acid group: D and E; (6) residues with a side chain that has an amide group: $\mathrm{Q}$ and $\mathrm{N}$; (7) residues with a side chain that has a positively charged group: $\mathrm{R}$ and $\mathrm{K}$; (8) $\mathrm{H}$; (9) P. Amino acid residues in the same category are considered to be matched. If three residues were matched in two interaction patterns, we then aligned them according to the order of the matched residues and calculated the RMSD value between them. Otherwise, these two interaction patterns were considered to be dissimilar. The RMSD value of two interaction patterns is calculated as the following equation:

$$
\operatorname{RMSD}=\sqrt{\frac{\sum_{i=1}^{n}\left(\left(x_{1, i}-x_{2, i}\right)^{2}+\left(y_{1, i}-y_{2, i}\right)^{2}+\left(z_{1, i}-z_{2, i}\right)^{2}\right)}{n}}
$$

Here, $x_{1, \mathrm{i}}, y_{1, \mathrm{i}}$ and $z_{1, \mathrm{i} ;} x_{2, \mathrm{i}}, y_{2, \mathrm{i}}$ and $z_{2, \mathrm{i}}$,refer to the coordinates of the $i$ th features in the two interaction patterns respectively. The features in each interaction pattern are defined as the mass center of the fragment and three residues. The interaction patterns that achieve both the fragment similarity and spatial similarity thresholds will be used to derive the CIPs. As the criteria defined in the manuscript, we selected the interaction patterns appeared in most of the protein-ligand complex structures as the CIPs. 
Table S1. Summary of the CIP comparisons from different protein-ligand complex structures for $\beta$-AR

\begin{tabular}{|c|c|c|c|c|c|c|c|c|c|c|c|c|c|c|c|c|c|c|c|c|c|c|c|c|c|c|c|c|c|c|}
\hline & P1 & P2 & P3 & P4 & P5 & P6 & P7 & P8 & P9 & P10 & P11 & P12 & P13 & P14 & P15 & P16 & P17 & P18 & P19 & P20 & P21 & P22 & $\mathbf{P 2 3}$ & P24 & P25 & P26 & P27 & P28 & P29 & P30 \\
\hline P1 & 2 & 1 & 0 & 0 & 0 & 0 & 1 & 2 & 1 & 2 & 1 & 2 & 1 & 1 & 2 & 1 & 1 & 1 & 1 & 1 & 1 & 1 & 2 & 1 & 1 & 1 & 1 & 2 & 2 & 2 \\
\hline P2 & 1 & 1 & 0 & 0 & 0 & 0 & 1 & 1 & 1 & 1 & 1 & 1 & 0 & 1 & 1 & 1 & 0 & 1 & 1 & 1 & 1 & 1 & 1 & 1 & 0 & 0 & 0 & 1 & 1 & 1 \\
\hline P3 & 0 & 0 & 2 & 0 & 0 & 0 & 0 & 0 & 0 & 0 & 0 & 0 & 0 & 0 & 0 & 0 & 0 & 0 & 0 & 0 & 0 & 0 & 0 & 0 & 0 & 0 & 0 & 0 & 0 & 0 \\
\hline P4 & 0 & 0 & 0 & 2 & 0 & 0 & 0 & 0 & 0 & 0 & 0 & 0 & 0 & 0 & 0 & 0 & 0 & 0 & 0 & 0 & 0 & 0 & 0 & 0 & 0 & 0 & 0 & 0 & 0 & 0 \\
\hline P5 & 0 & 0 & 0 & 0 & 1 & 1 & 0 & 0 & 0 & 0 & 0 & 0 & 0 & 0 & 0 & 0 & 1 & 0 & 0 & 0 & 0 & 0 & 0 & 0 & 0 & 1 & 1 & 0 & 0 & 0 \\
\hline P6 & 0 & 0 & 0 & 0 & 1 & 2 & 0 & 0 & 0 & 0 & 0 & 0 & 0 & 0 & 0 & 1 & 1 & 1 & 0 & 0 & 0 & 0 & 0 & 0 & 1 & 1 & 2 & 0 & 0 & 0 \\
\hline P7 & 1 & 1 & 0 & 0 & 0 & 0 & 1 & 1 & 1 & 1 & 1 & 1 & 0 & 1 & 1 & 1 & 0 & 1 & 1 & 1 & 1 & 1 & 1 & 1 & 0 & 0 & 0 & 1 & 1 & 1 \\
\hline P8 & 2 & 1 & 0 & 0 & 0 & 0 & 1 & 2 & 1 & 2 & 1 & 2 & 1 & 2 & 2 & 1 & 1 & 1 & 1 & 1 & 1 & 1 & 2 & 1 & 1 & 1 & 1 & 2 & 2 & 2 \\
\hline P9 & 1 & 1 & 0 & 0 & 0 & 0 & 1 & 1 & 1 & 1 & 1 & 1 & 0 & 1 & 1 & 1 & 0 & 1 & 1 & 1 & 1 & 1 & 1 & 1 & 0 & 0 & 0 & 1 & 1 & 1 \\
\hline P10 & 2 & 1 & 0 & 0 & 0 & 0 & 1 & 2 & 1 & 2 & 1 & 2 & 1 & 2 & 2 & 1 & 1 & 1 & 1 & 1 & 1 & 1 & 2 & 1 & 1 & 1 & 1 & 2 & 2 & 2 \\
\hline P11 & 1 & 1 & 0 & 0 & 0 & 0 & 1 & 1 & 1 & 1 & 1 & 1 & 0 & 1 & 1 & 1 & 0 & 1 & 1 & 1 & 1 & 1 & 1 & 1 & 0 & 0 & 0 & 1 & 1 & 1 \\
\hline P12 & 2 & 1 & 0 & 0 & 0 & 0 & 1 & 2 & 1 & 2 & 1 & 2 & 1 & 2 & 2 & 1 & 1 & 1 & 1 & 1 & 1 & 1 & 2 & 1 & 0 & 1 & 0 & 2 & 2 & 2 \\
\hline P13 & 1 & 0 & 0 & 0 & 0 & 0 & 0 & 1 & 0 & 1 & 0 & 1 & 2 & 1 & 1 & 0 & 1 & 0 & 0 & 0 & 0 & 0 & 1 & 0 & 0 & 1 & 0 & 1 & 1 & 1 \\
\hline P14 & 2 & 1 & 0 & 0 & 0 & 0 & 1 & 2 & 1 & 2 & 1 & 2 & 1 & 2 & 2 & 1 & 1 & 1 & 1 & 1 & 1 & 1 & 2 & 1 & 0 & 1 & 0 & 2 & 2 & 2 \\
\hline P15 & 2 & 1 & 0 & 0 & 0 & 0 & 1 & 2 & 1 & 2 & 1 & 2 & 1 & 2 & 2 & 1 & 1 & 1 & 1 & 1 & 1 & 1 & 2 & 1 & 0 & 1 & 0 & 2 & 2 & 2 \\
\hline P16 & 1 & 1 & 0 & 0 & 0 & 1 & 1 & 1 & 1 & 1 & 1 & 1 & 0 & 1 & 1 & 2 & 0 & 2 & 1 & 1 & 1 & 1 & 2 & 1 & 1 & 0 & 1 & 1 & 1 & 1 \\
\hline P17 & 1 & 0 & 0 & 0 & 1 & 1 & 0 & 1 & 0 & 1 & 0 & 1 & 1 & 1 & 1 & 0 & 2 & 0 & 0 & 0 & 0 & 0 & 1 & 0 & 1 & 2 & 2 & 1 & 1 & 1 \\
\hline P18 & 1 & 1 & 0 & 0 & 0 & 1 & 1 & 1 & 1 & 1 & 1 & 1 & 0 & 1 & 1 & 2 & 0 & 2 & 1 & 1 & 1 & 1 & 1 & 1 & 1 & 0 & 1 & 2 & 1 & 1 \\
\hline P19 & 1 & 1 & 0 & 0 & 0 & 0 & 1 & 1 & 1 & 1 & 1 & 1 & 0 & 1 & 1 & 1 & 0 & 1 & 1 & 1 & 1 & 1 & 1 & 1 & 0 & 0 & 0 & 1 & 1 & 1 \\
\hline P20 & 1 & 1 & 0 & 0 & 0 & 0 & 1 & 1 & 1 & 1 & 1 & 1 & 0 & 1 & 1 & 1 & 0 & 1 & 1 & 1 & 1 & 1 & 1 & 1 & 0 & 0 & 0 & 1 & 1 & 1 \\
\hline P21 & 1 & 1 & 0 & 0 & 0 & 0 & 1 & 1 & 1 & 1 & 1 & 1 & 0 & 1 & 1 & 1 & 0 & 1 & 1 & 1 & 1 & 1 & 1 & 1 & 0 & 0 & 0 & 1 & 1 & 1 \\
\hline P22 & 1 & 1 & 0 & 0 & 0 & 0 & 1 & 1 & 1 & 1 & 1 & 1 & 0 & 1 & 1 & 1 & 0 & 1 & 1 & 1 & 1 & 1 & 1 & 1 & 0 & 0 & 0 & 1 & 1 & 1 \\
\hline $\mathbf{P 2 3}$ & 2 & 1 & 0 & 0 & 0 & 0 & 1 & 2 & 1 & 2 & 1 & 2 & 1 & 2 & 2 & 1 & 1 & 1 & 1 & 1 & 1 & 1 & 2 & 1 & 1 & 1 & 1 & 2 & 2 & 2 \\
\hline P24 & 1 & 1 & 0 & 0 & 0 & 0 & 1 & 1 & 1 & 1 & 1 & 1 & 0 & 1 & 1 & 1 & 0 & 1 & 1 & 1 & 1 & 1 & 1 & 1 & 0 & 0 & 0 & 1 & 1 & 1 \\
\hline P25 & 1 & 0 & 0 & 0 & 0 & 1 & 0 & 1 & 0 & 1 & 0 & 0 & 0 & 0 & 0 & 1 & 1 & 1 & 0 & 0 & 0 & 0 & 1 & 0 & 1 & 0 & 1 & 1 & 1 & 1 \\
\hline P26 & 1 & 0 & 0 & 0 & 1 & 1 & 0 & 1 & 0 & 1 & 0 & 1 & 1 & 1 & 1 & 0 & 2 & 0 & 0 & 0 & 0 & 0 & 1 & 0 & 0 & 1 & 0 & 1 & 1 & 1 \\
\hline P27 & 1 & 0 & 0 & 0 & 1 & 2 & 0 & 1 & 0 & 1 & 0 & 0 & 0 & 0 & 0 & 1 & 2 & 1 & 0 & 0 & 0 & 0 & 1 & 0 & 1 & 0 & 1 & 1 & 1 & 1 \\
\hline P28 & 2 & 1 & 0 & 0 & 0 & 0 & 1 & 2 & 1 & 2 & 1 & 2 & 1 & 2 & 2 & 1 & 1 & 2 & 1 & 1 & 1 & 1 & 2 & 1 & 1 & 1 & 1 & 2 & 2 & 2 \\
\hline P29 & 2 & 1 & 0 & 0 & 0 & 0 & 1 & 2 & 1 & 2 & 1 & 2 & 1 & 2 & 2 & 1 & 1 & 1 & 1 & 1 & 1 & 1 & 2 & 1 & 1 & 1 & 1 & 2 & 2 & 2 \\
\hline
\end{tabular}


The PDB codes of the protein structures for P1-P30:

P1: 2RH1, P2: 2VT4, P3: 2Y00, P4: 2Y01, P5: 2Y02, P6: 2Y03, P7: 2Y04, P8: 2YCW, P9: 2YCX, P10: 2YCY, P11: 2YCZ, P12: 3D4S, P13: 3NY8, P14: 3NY9, P15: 3NYA, P16: 3P0G: P17: 3PDS, P18: 3SN6, P19: 3ZPQ, P20: 3ZPR, P21: 4AMI, P22: 4AMJ, P23: 4BVN, P24: 4LDE, P25: 4LDL, P26: 4LDO, P27: 4QKX,

P28: 5A8E, P29: 5F8U, P30: 5JQH

Table S2. Summary of the CIP comparisons from different protein-ligand complex structures for mAChR

\begin{tabular}{|c|c|c|c|c|c|c|c|c|c|}
\hline & 3UON & 4DAJ & 4MQS & 4MQT & 4U14 & 4U15 & 4U16 & 5CXV & 5DSG \\
\hline 3UON & 3 & 2 & 0 & 0 & 2 & 3 & 1 & 3 & 3 \\
\hline 4DAJ & 2 & 1 & 0 & 0 & 1 & 2 & 0 & 2 & 2 \\
\hline 4MQS & 0 & 0 & 1 & 1 & 0 & 0 & 0 & 0 & 0 \\
\hline 4MQT & 0 & 0 & 1 & 1 & 0 & 0 & 0 & 0 & 0 \\
\hline 4U14 & 2 & 1 & 0 & 0 & 2 & 2 & 1 & 1 & 2 \\
\hline 4U15 & 3 & 2 & 0 & 0 & 2 & 3 & 1 & 2 & 3 \\
\hline 4U16 & 1 & 0 & 0 & 0 & 1 & 1 & 1 & 0 & 1 \\
\hline 5CXV & 3 & 2 & 0 & 0 & 1 & 2 & 0 & 3 & 2 \\
\hline 5DSG & 3 & 2 & 0 & 0 & 2 & 3 & 1 & 2 & 3 \\
\hline
\end{tabular}

Table S1 and S2 summarize the results of CIP similarity comparisons between different protein-ligand complexes. The digit in the table indicates the number of matched CIPs between two protein-ligand complexes. 


\section{Preparation of fragment-residue interaction pattern library}

The GPCR ligands used in this study for generating library of fragment-residue interaction pattern were collected from two public databases, i.e. GPCRSARfari (ftp://ftp.ebi.ac.uk/pub/databases/chembl/GPCRSARfari/) and DrugBank (https://www.drugbank.ca/). The 2D SDF files of GPCR ligands were downloaded from the websites. They were prepared as the standard structure files by a series of routine operations. The 2D files were imported into Canvas module in Schrödinger package (version 2017, Schrödinger LLC., USA) with the duplicate structures removed. The molecules with molecular weight above 800 were also removed since they might be antibodies, polysaccharides or large peptides. Each molecule was minimized to keep a low energy conformation for molecular docking.

To save the computational costs, one protein structure was selected for molecular docking to represent the two GPCR family proteins, i.e. 2RH1 for $\beta$-AR and 4U15 for mAChR. The molecular docking was performed by the program GOLD (version 5.2, Cambridge Crystallographic Data Centre, UK) with scoring function ChemPLP. The binding site was defined as the residues within $6 \AA$ of the bound ligand. The conformation sampling efficiency was set as $100 \%$. The GA number was set as 10 and the top three conformations were kept for each GPCR ligand. Other GA parameters were set as "auto". The docking conformations of all GPCR ligands were cut into fragments to generate interaction patterns with the nearest three residues, which formed the fragment-interaction pattern library. 


\section{PART 2. Bioassay details}

\section{1. cAMP assay for $\beta_{2}-A R$}

Stable cell pools that overexpress human $\beta_{2}$-AR in HEK293F background were generated in Amgen Asia R\&D Center. Cells were cultured in FreeStyle 293 Expression Medium (Life, Cat.\#12338026) supplemented with $1.6 \mu \mathrm{g} / \mathrm{mL}$ puromycin (Life, Cat.\# A1113803). Activation or inhibition of $\beta_{2}$-ARby different small molecular compounds was measured based on intracellular cAMP levels using Lance Ultra cAMP kit (PerkinElmer) according to manufacturer's protocol. In agonist mode, in a well of 384-well plate, 1,000 cells in $5 \mu \mathrm{l}$ assay buffer (HBSS buffer pH7.4 with 5 $\mathrm{mM}$ HEPES, $0.1 \%$ BSA and $0.5 \mathrm{mM}$ IBMX) were mixed with $5 \mu \mathrm{l}$ of compound in assay buffer, incubated for $30 \mathrm{~min}$ at $37^{\circ} \mathrm{C}$; in antagonist mode, 1,000 cells in $5 \mu 1$ assay buffer were mixed with $2.5 \mu \mathrm{l}$ of compound in assay buffer, incubated for 15 min at $37^{\circ} \mathrm{C}$, then mixed with $2.5 \mu \mathrm{L}$ of $4 \times \mathrm{EC}_{80}$ of agonist (isoproterenol) in assay buffer and incubated for $30 \mathrm{~min}$ at $37^{\circ} \mathrm{C}$. After incubation, $5 \mu \mathrm{L}$ of $4 \times$ Eu-cAMP and 5 $\mu \mathrm{L}$ of $4 \times$ Ulight-Anti-cAMP working solutions were added to each well and incubated at RT for 60 mins before read with Envision (PerkinElmer). All signals (ratio of $665 \mathrm{~nm} / 615 \mathrm{~nm}$ ) were normalized to positive and negative control, then fit with a sigmoidal dose-response model using GraphPad Prism software to determine $E C_{50}$ or $I C_{50}$ of compounds.

\section{Aequorin luminescent cellular calcium assay for M1}

Human Muscarinic Acetylcholine Receptor M1 Aequorin cells on CHO-K1 background was purchased from Perkin Elmer (Cat.\# ES-210-A) . Cells were cultured in DMEM/F12 medium supplemented with 10\% FBS, 1\% Penicillin/ Streptomycin (Life Tech, Cat.\# 15140122), 0.4 mg/mL Geneticin (Life, Cat.\#10131035), 0.25 $\mathrm{mg} / \mathrm{mL}$ Zeocin (Life, Cat.\# R25001). Functional calcium aequorin assay was performed to evaluate the activation or inhibition of M1 receptor by different small molecular compounds. After cells were dissociated by trypsin-EDTA and resuspended in assay buffer (DMEM/F12 without phenol red, $0.1 \%$ BSA) to a concentration of $5 \times 10^{5} / \mathrm{mL}$, Coelenterazine was loaded into cells by adding Coelenterazine to a final 
concentration of $5 \mu \mathrm{M}$ to the cell suspension and incubated at room temperature for 4 hours. Before compound treatment, $20 \mu \mathrm{L}$ of cells were dispensed into a well in a 384 well plate. Then $20 \mu \mathrm{L}$ of compound diluted with assay buffer was injected into well using FLIRPtetra and luminescence signal was recorded simultaneously to detect signal stimulated by any potential agonist. Cells were treated by compound at RT for 20 minutes then $20 \mu \mathrm{L}$ of $3 \times \mathrm{EC}_{80}$ agonist (Acetylcholine) was injected to well using FLIRPtetra and luminescence signal was also recorded simultaneously as signal from "antagonist". The area under curve was calculated, normalized to positive and negative control, then plotted against logarithm of compound concentration to determine $E C_{50}$ or $I C_{50}$ of compound with a sigmoidal dose-response model using GraphPad Prism 7 software. 


\section{PART 3. MD simulations and MM-GB/SA calculation}

The 3D structures of candidate compounds were sketched and minimized with MOE software (version 2016, Chemical Computing Group, https://www.chemcomp.com/Products.htm). For $\beta_{2}$-AR, the receptor used for docking was selected from the crystal structure formed by $\beta_{2}$-AR and its antagonist carazolol (PDB code: 2RH1). For M1, the receptor used for docking was selected form the crystal structure formed by M1 and its antagonist tiotropium (PDB code: 5CXV). The molecular docking task was run by the program GOLD (version 5.2, Cambridge Crystallographic Data Center) with scoring function ChemPLP. The binding site was defined by residues within $6 \AA$ of the reference ligands in the crystal structures. Early termination was prohibited. Other parameters were set as default. A total of 30 docking conformations were generated and clustered by RMSD values with a cutoff of $2.0 \AA$ A. A reasonable conformation was finally picked out from the best-scored docking conformations of all clusters after visual examination. For comparisons, the positive control compounds used in the bioassay tests were also docked with the receptors.

The selected docking conformations of candidate compounds and control compounds were then refined through MD simulation by using AMBER program (version 14, University of California San Francisco). To set up the MD simulations, the model of GPCR-ligand complex structure soaked in the phospholipid bilayer membrane environment was built via the online server CHARMM-GUI (www.charmm-gui.org/?doc=input/membrane). POPC bilayer membrane was selected in this step. The number of water molecules per one lipid molecule was set as 37 . The number of lipids was adjusted manually according to the results of the prompt calculation. After that, $0.15 \mathrm{M} \mathrm{KCl}$ was added to neutralize the system. Monte Carlo algorithm was used to place the ions. Then, the small-molecule ligand was applied with the GAFF force field, while the protein and phospholipid were applied with FF12SB force field. Bcc partial charges were calculated and assigned on the small-molecule ligand. The protein and lipid atoms were assigned the PARM10 template charges implemented in AMBER, and all ionizable residues were set at the default protonation states at the neutral $\mathrm{pH}$.

The prepared GPCR-ligand-POPC complex structures were first relaxed by 1000 
cycles of steepest descent minimization, followed by 9000 cycles of conjugated gradient minimization, with restraints on the heavy atoms of the protein and the ligand (restraint_wt $=10.0 \mathrm{kcal} \mathrm{mol}^{-1} \AA^{-2}$ ). Then, the complex structures were relaxed without restraint by 1000 cycles of steepest descent minimization, followed by 9000 cycles of conjugated gradient minimization. After that, the systems were firstly gradually heated up with the Berendsen algorithm from $0 \mathrm{~K}$ to $300 \mathrm{~K}$ in $500 \mathrm{ps}$ with restraints on the heavy atoms of the protein and the ligand (restraint_wt $=10.0 \mathrm{kcal} \mathrm{mol}^{-1} \AA^{-2}$ ). Then, the systems were equilibrated at $300 \mathrm{~K}$ for 500 ps. Finally, $100 \mathrm{~ns}$ long production was performed for each complex at a constant temperature of $300 \mathrm{~K}$ and a constant pressure of 1 atm. Electrostatic interactions were calculated with the PME algorithm. The distance cutoff of non-bonded interactions was set as $12 \AA$. The SHAKE algorithm was applied to fix the lengths of all chemical bonds connecting hydrogen atoms. All MD simulations were performed on a GPU server with 2 NIVIDIA Tesla P100 cards.

The last 50ns of the production trajectory was sampled each 10ps (a total of 5000 conformations) for MM-GB/SA calculation and energy decomposition analysis. MM-PBSA.py.MPI was used to perform the parallel computation with $8 \mathrm{CPU}$ cores on an Intel Xeon 5345-basedLinux cluster.
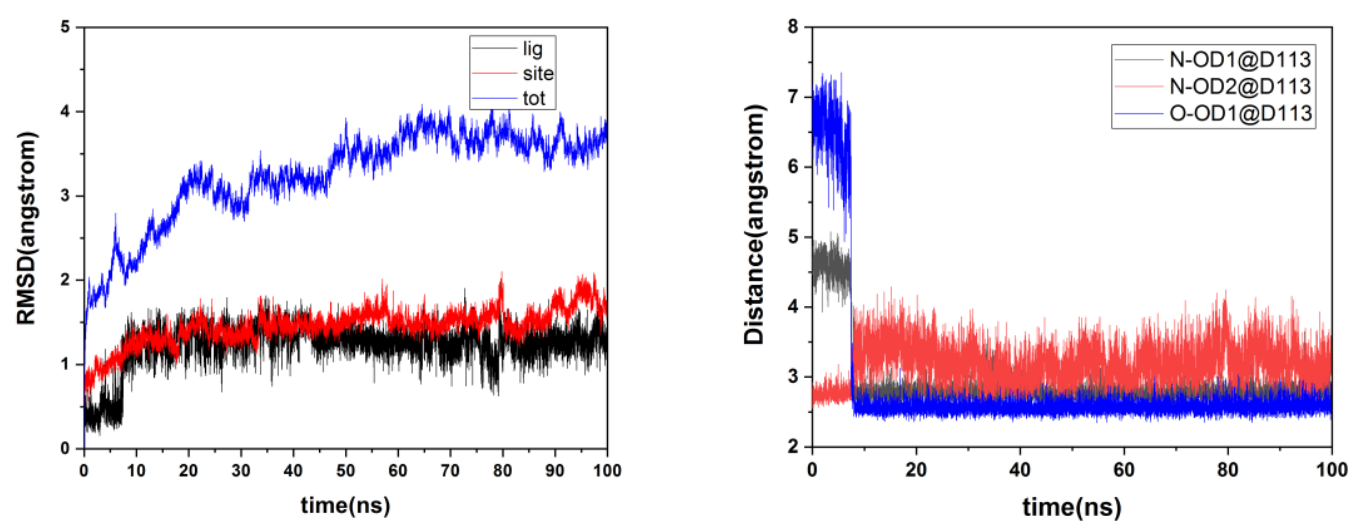

(a) 

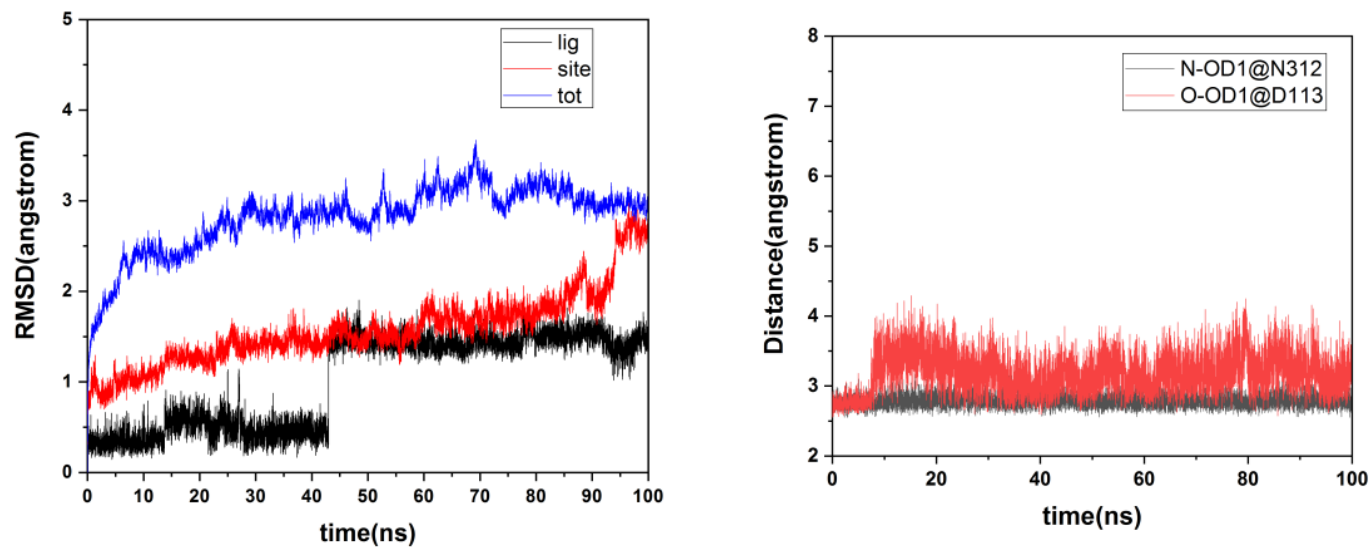

(b)
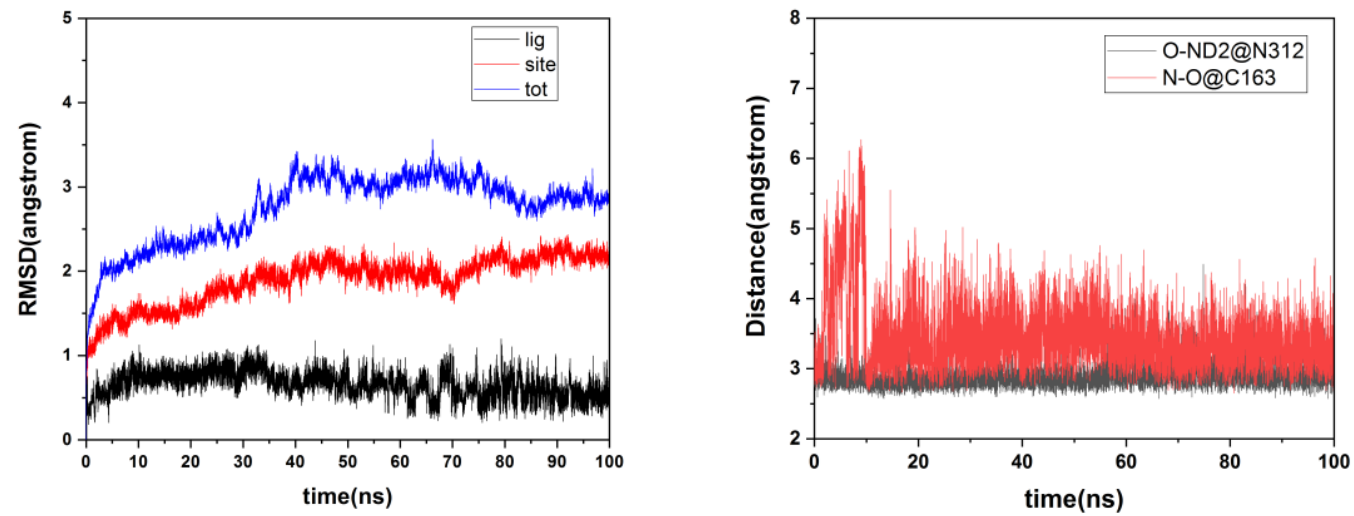

(c)

Figure S1. The MD simulation results of the $\beta_{2}-\mathrm{AR}$ protein complexed with three compounds (a) an inverse agonist ICI118551 (b) AS-06 and (c) AS-34. For each compound, the left panel depicts the conformational changes of the ligand, key residues of the binding pocket and the complex during the MD simulation. The right panel detects the distances of atom pairs which form the key hydrogen bond interactions between the protein and the ligand during the MD simulation.
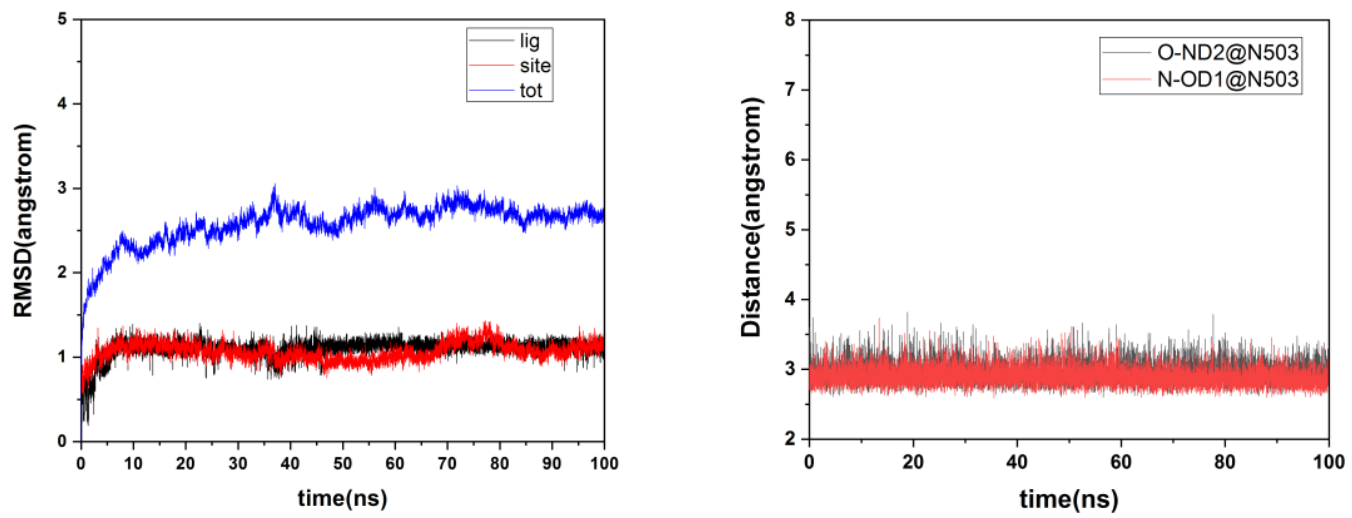

(a) 

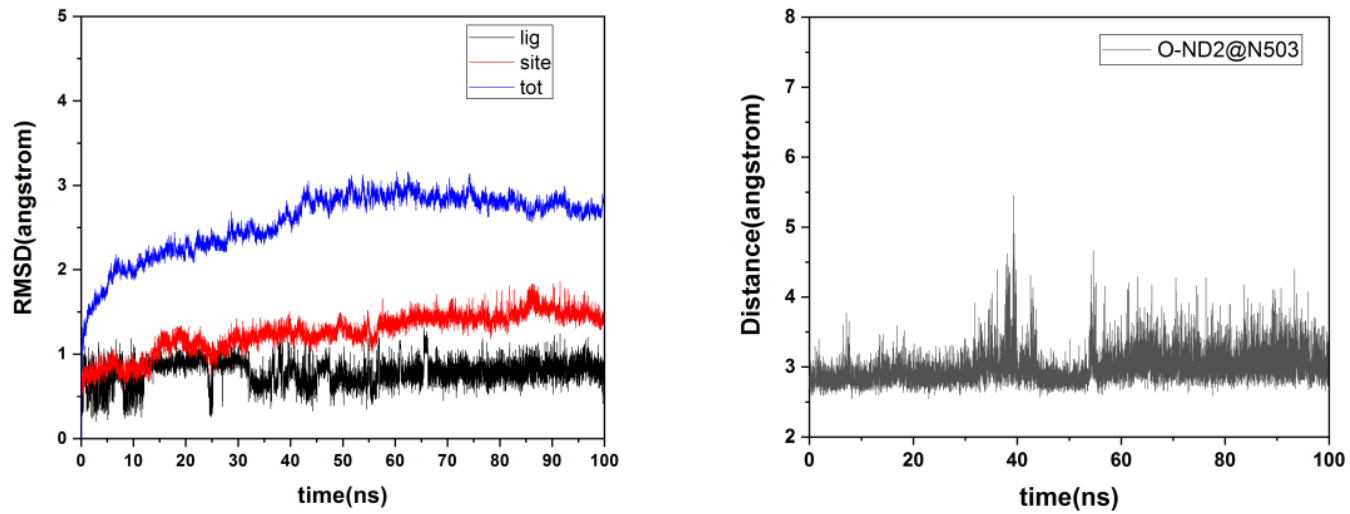

(b)
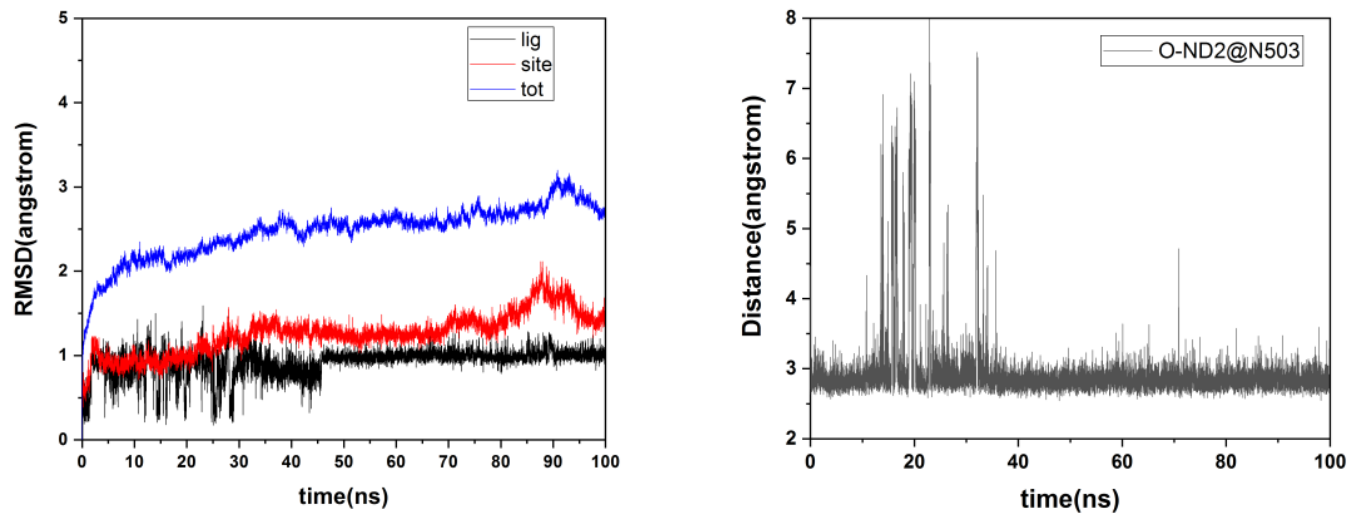

(c)

Figure S2. The MD simulation results of the $\beta_{2}$-AR protein complexed with three compounds (a) pirenzepine (b) AS-53 and (c) AS-56. For each compound, the left panel depicts the conformational changes of the ligand, key residues of the binding pocket and the complex during the MD simulation. The right panel detects the distances of atom pairs which form the key hydrogen bond interactions between the protein and the ligand during the MD simulation. 


\section{PART 4. Summary of the candidate compounds for biological test}

Table S3. Chemical structures, compound IDs, hypothetical target and dose response curves of 63 candidate compounds

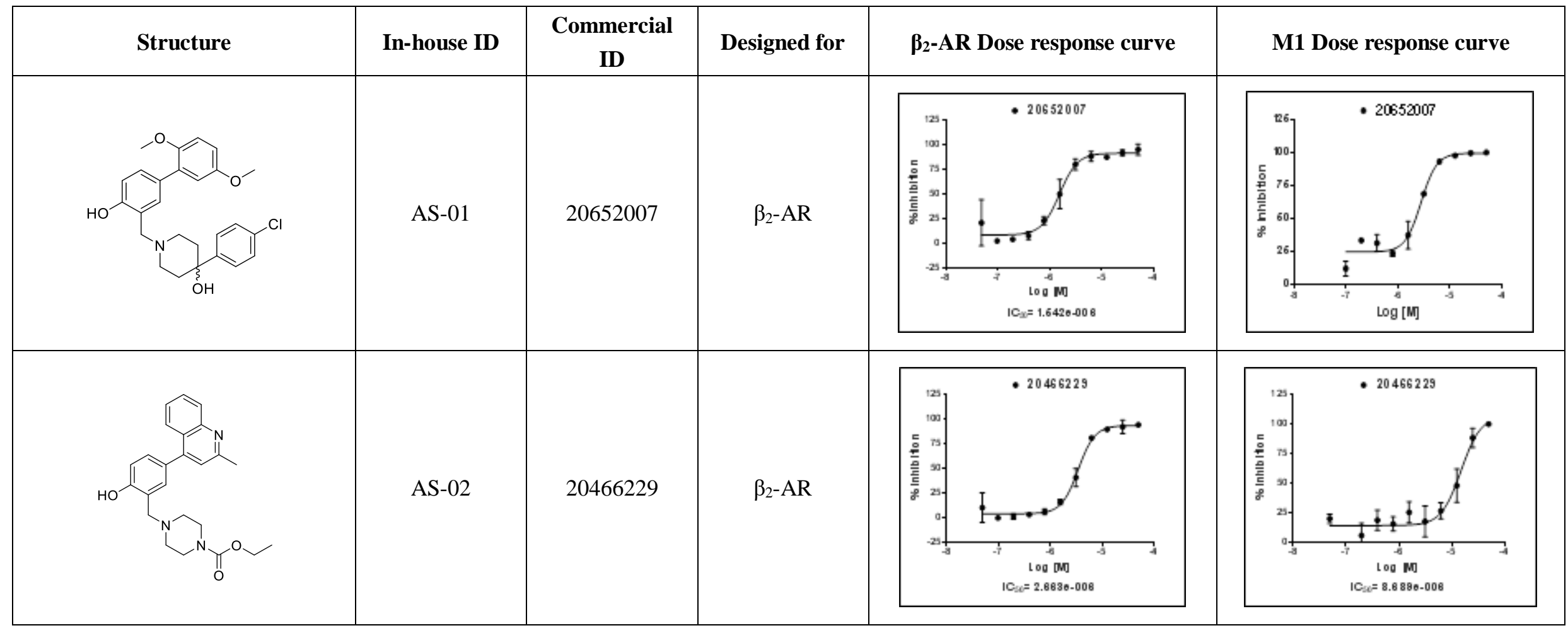




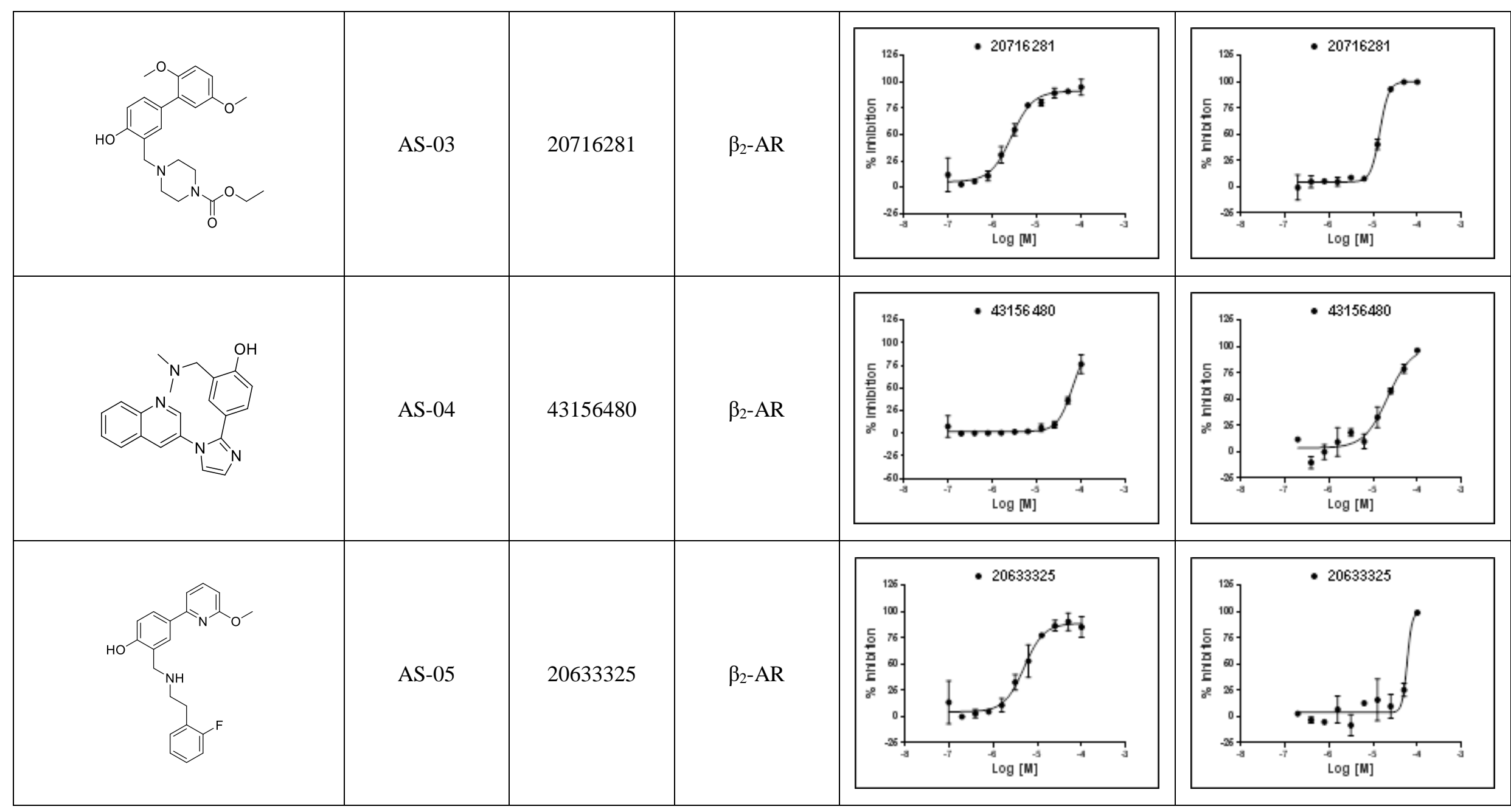




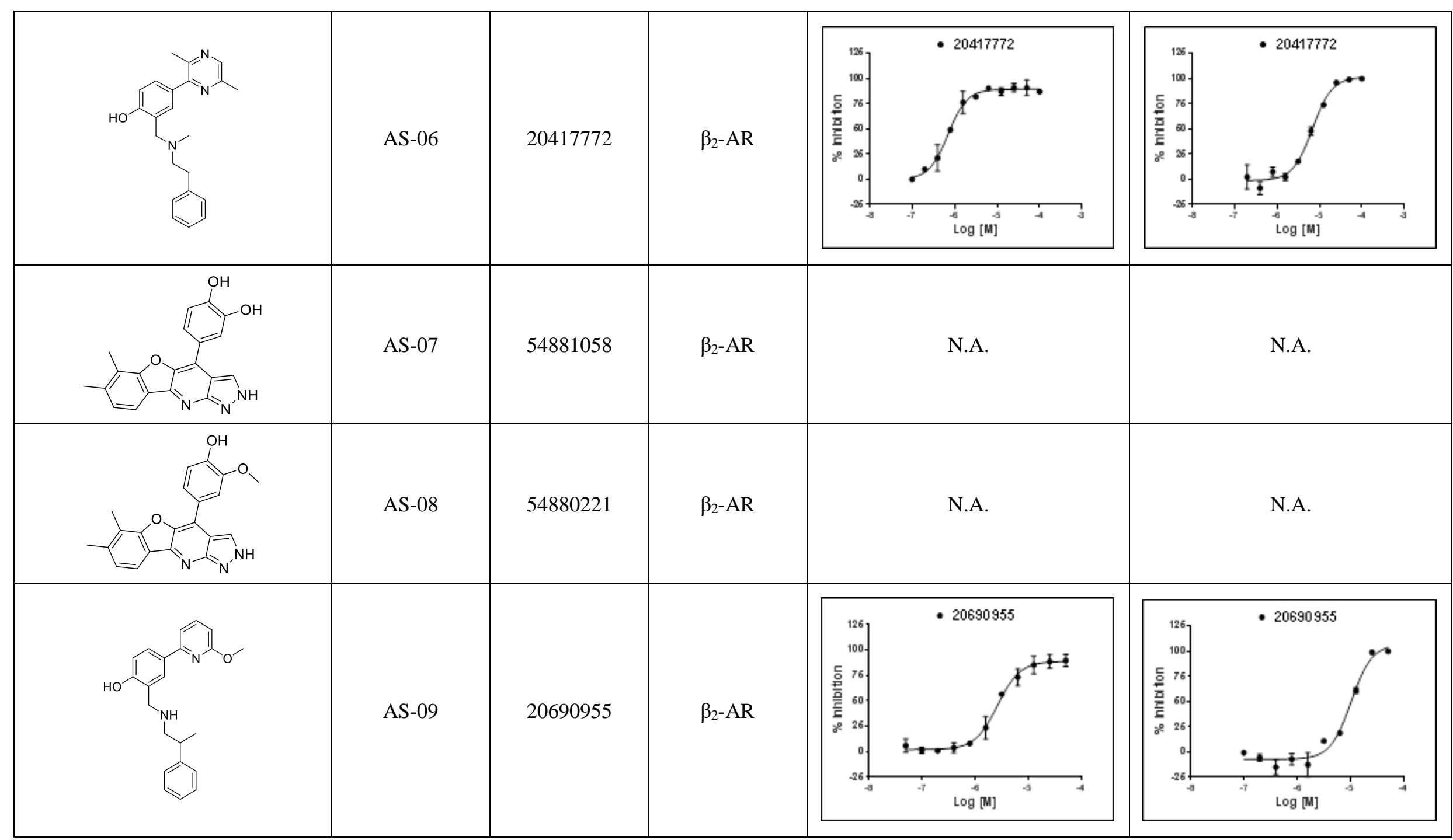




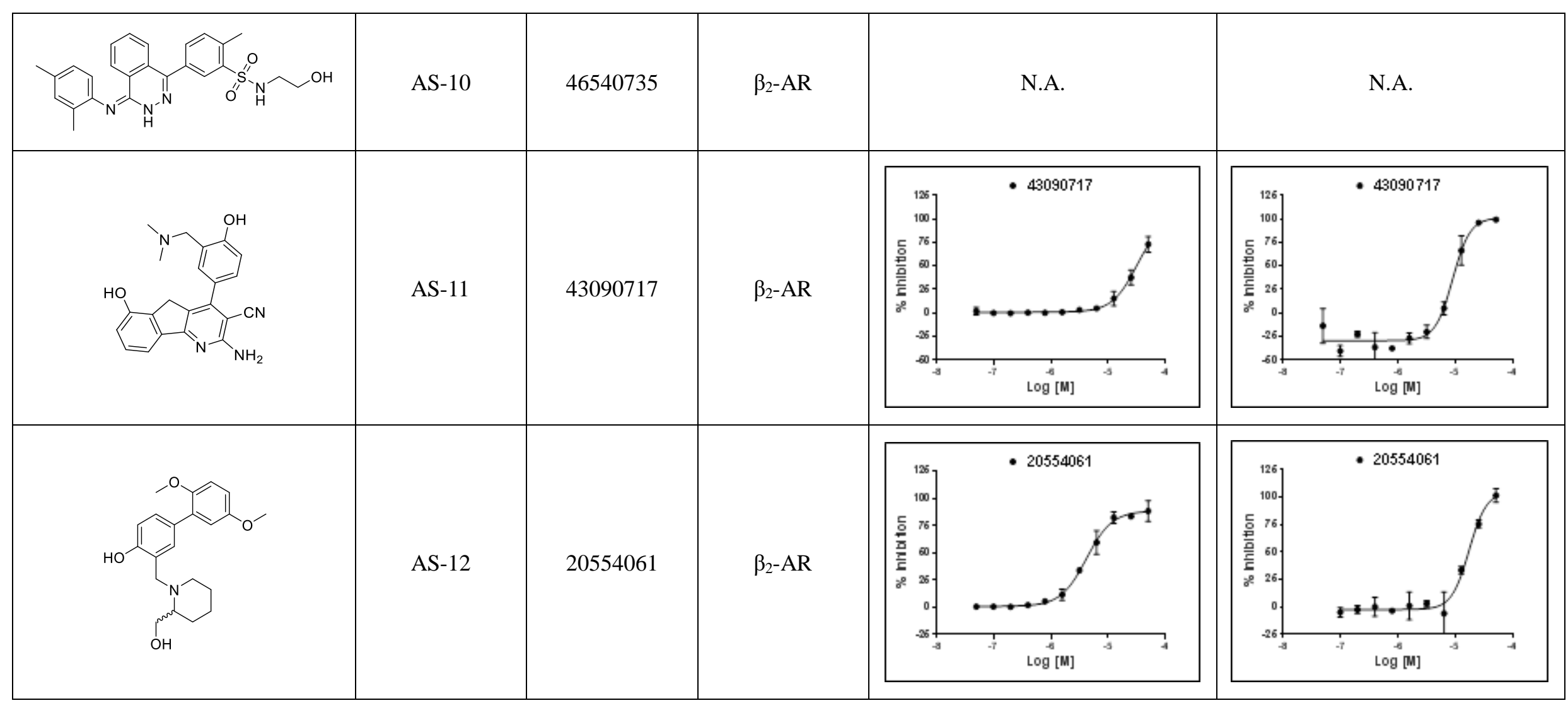




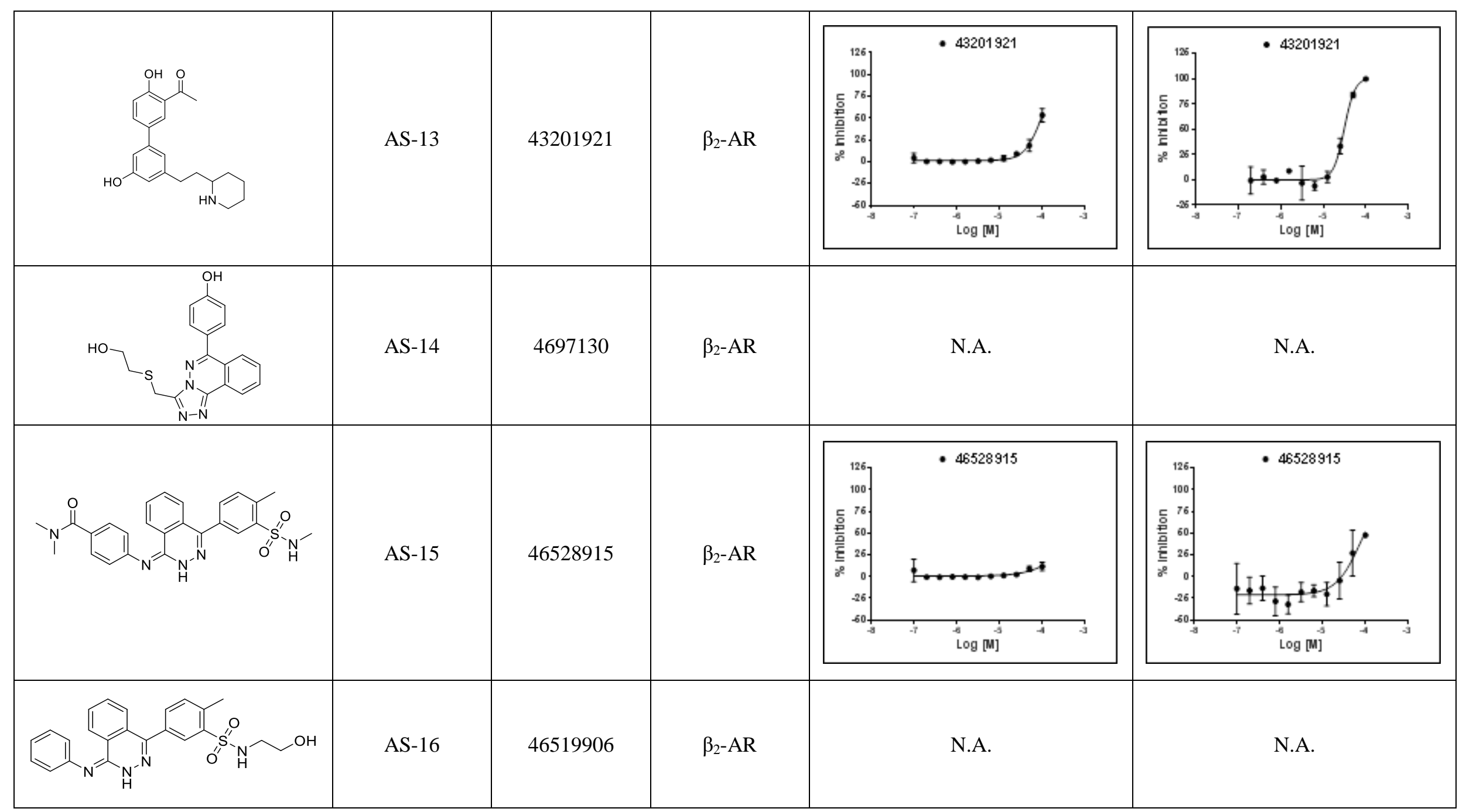




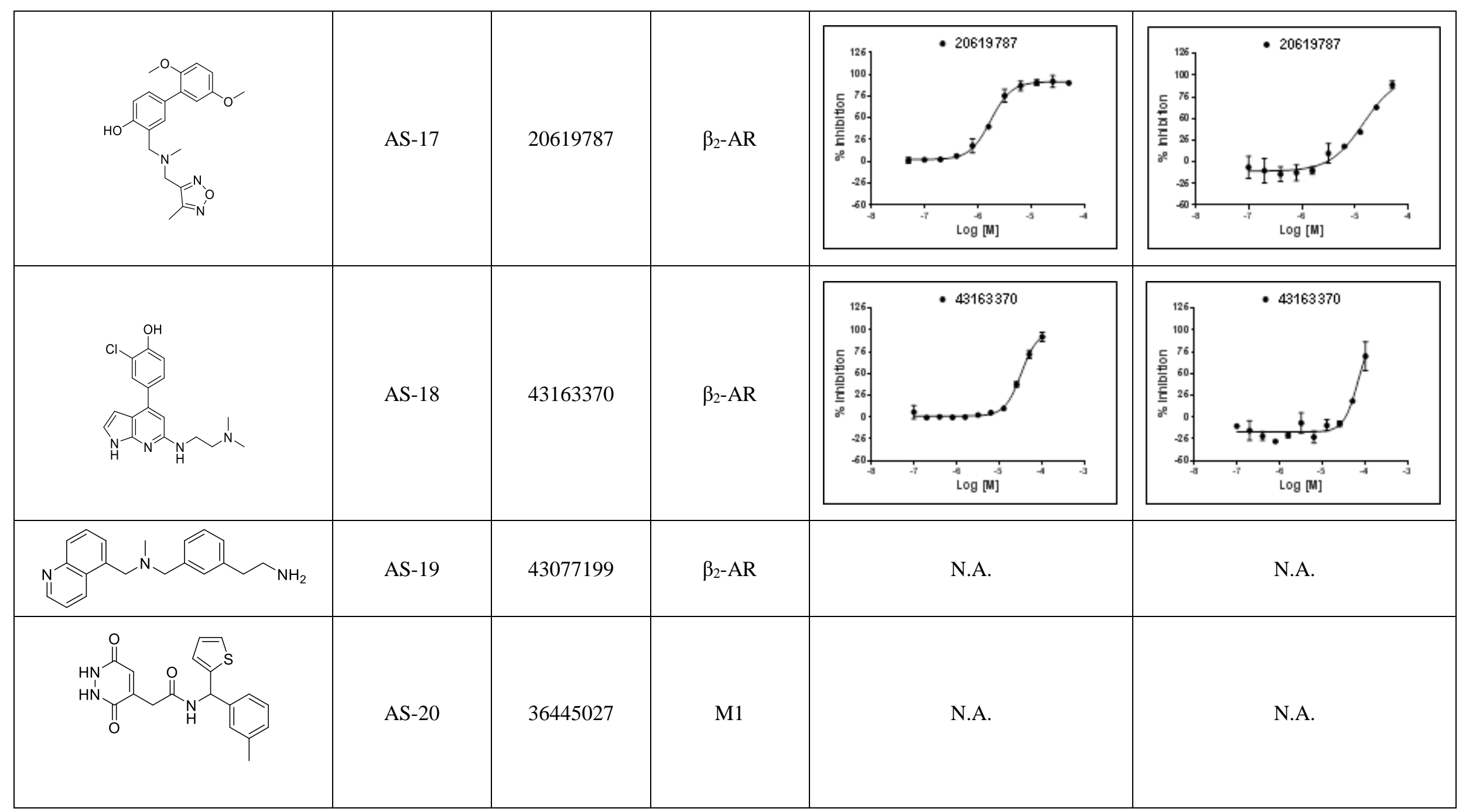




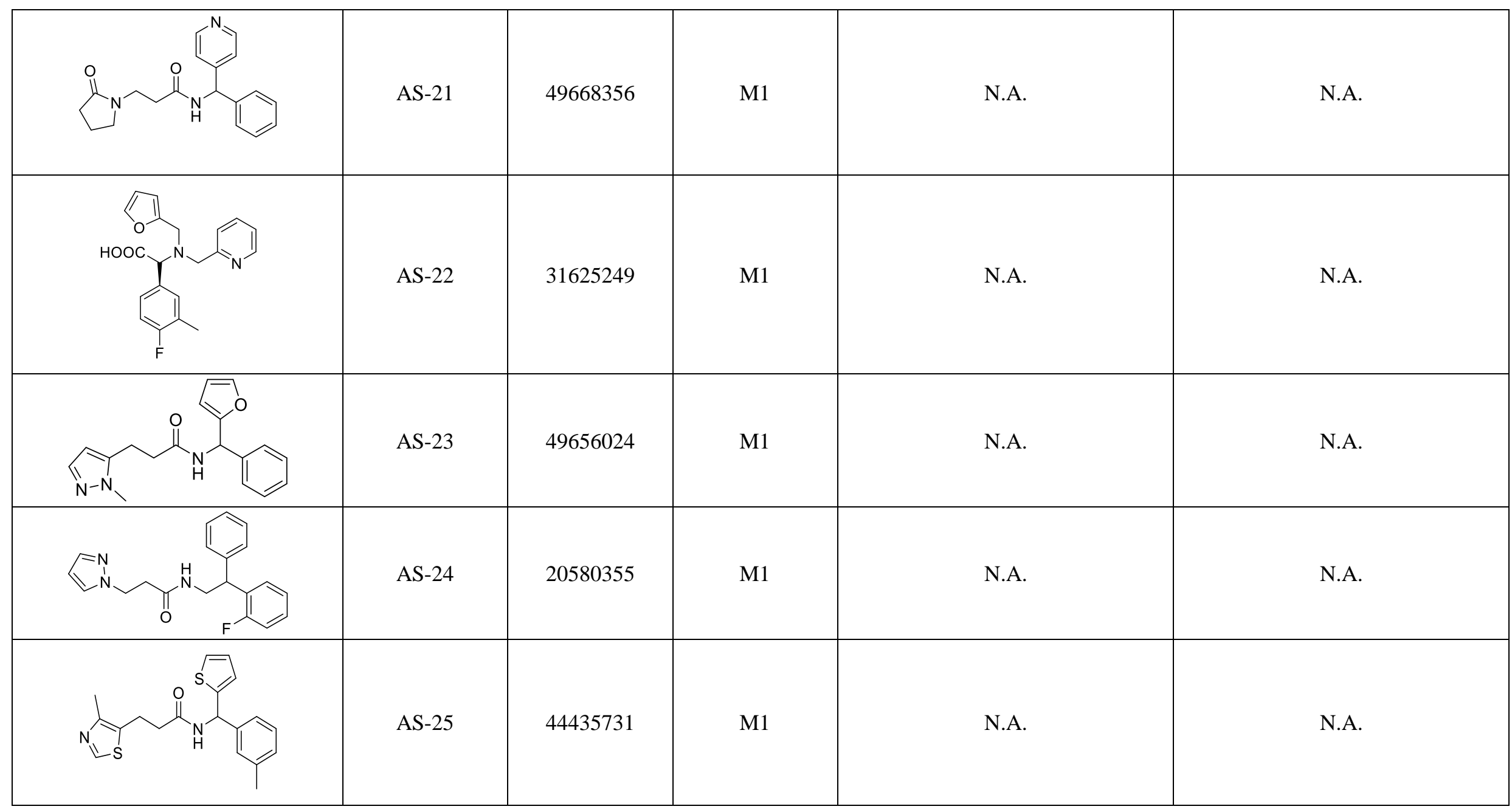




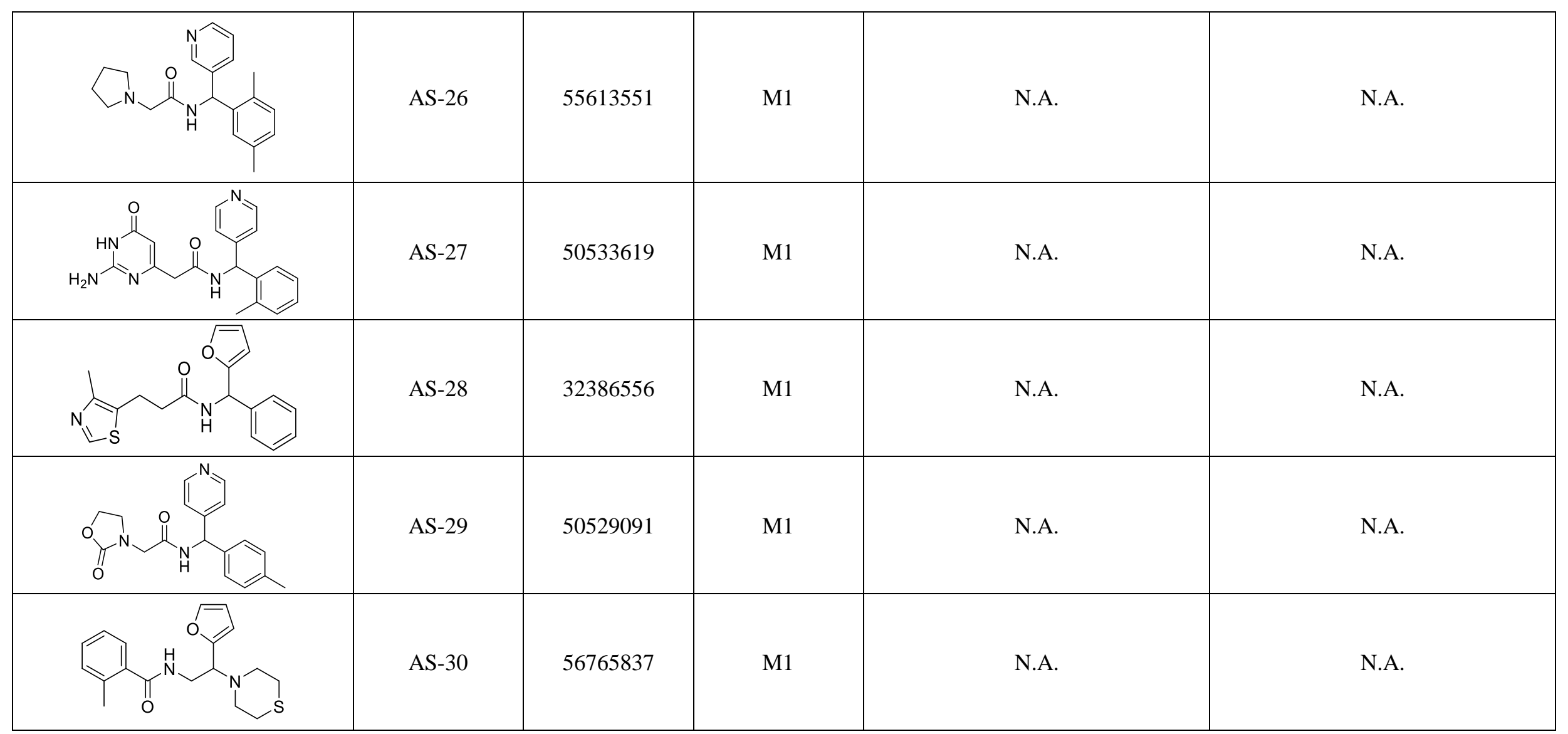




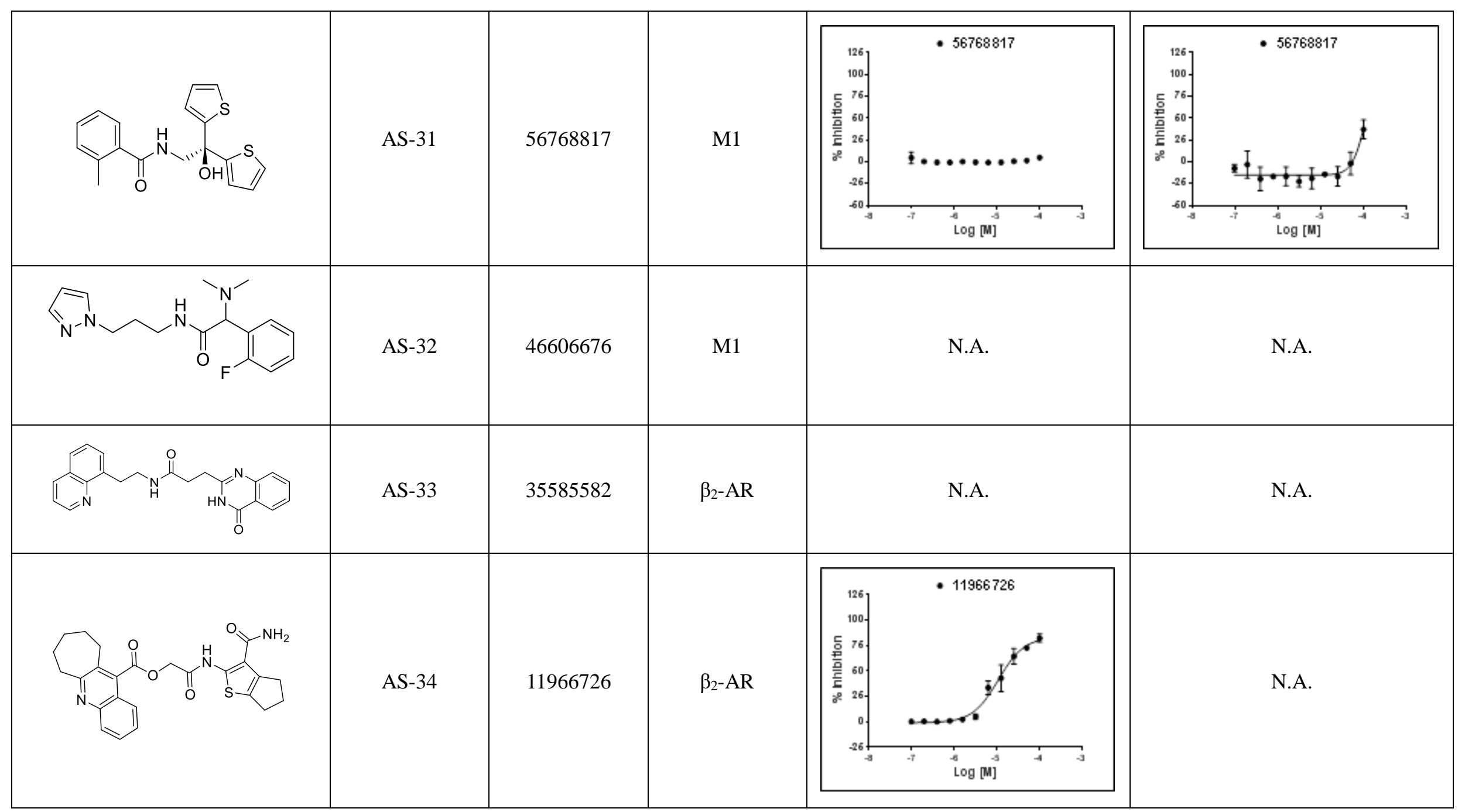




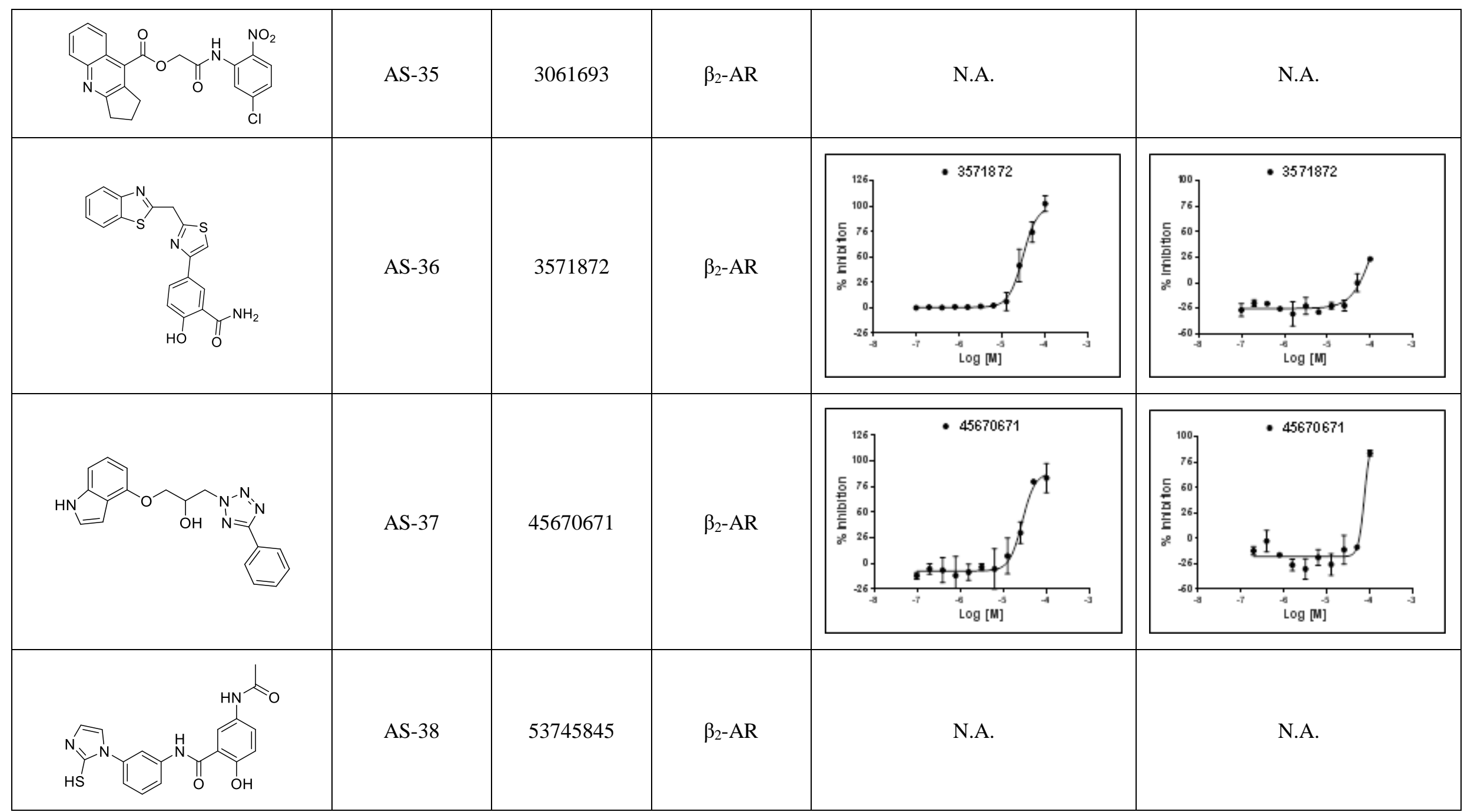




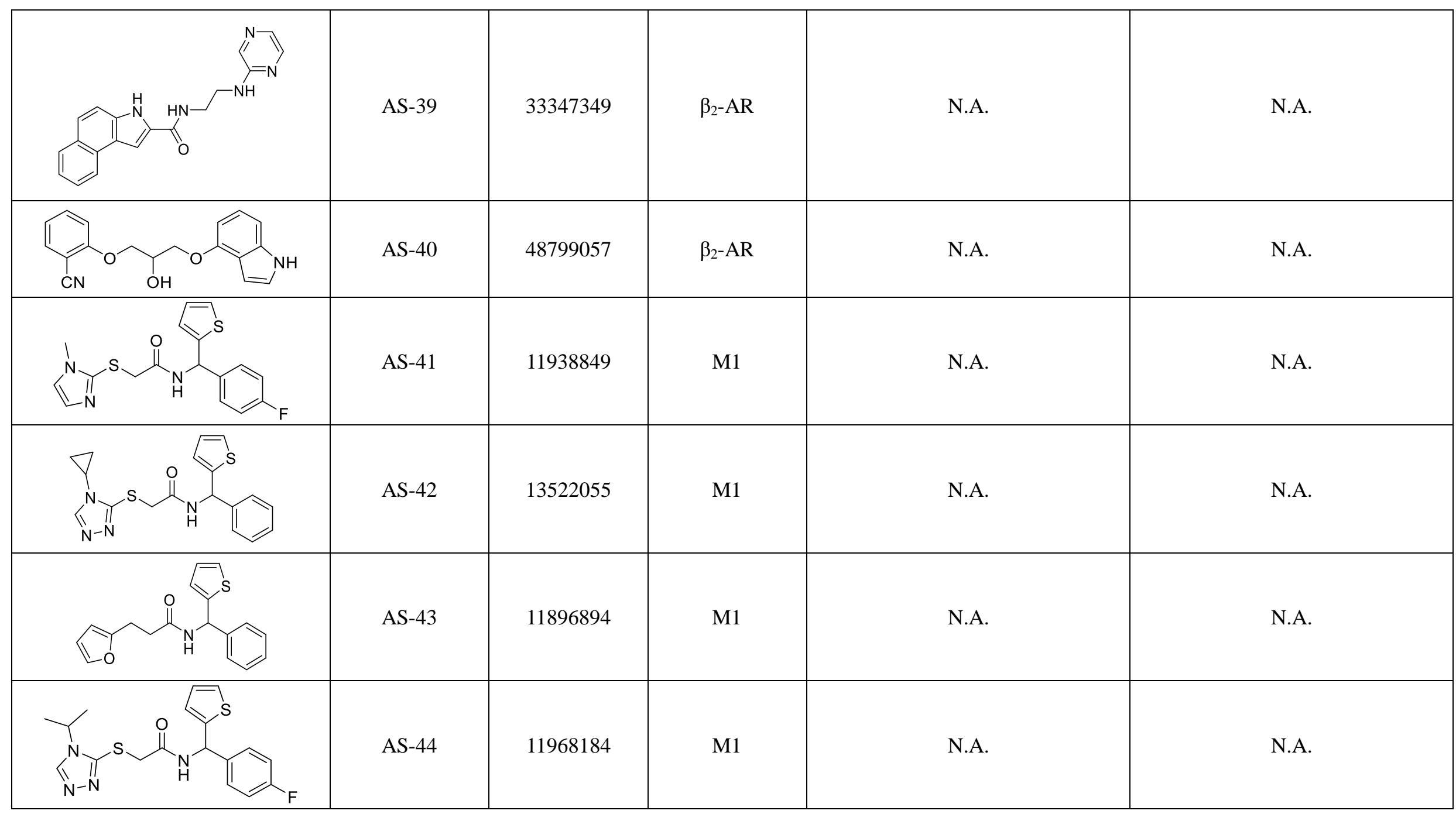




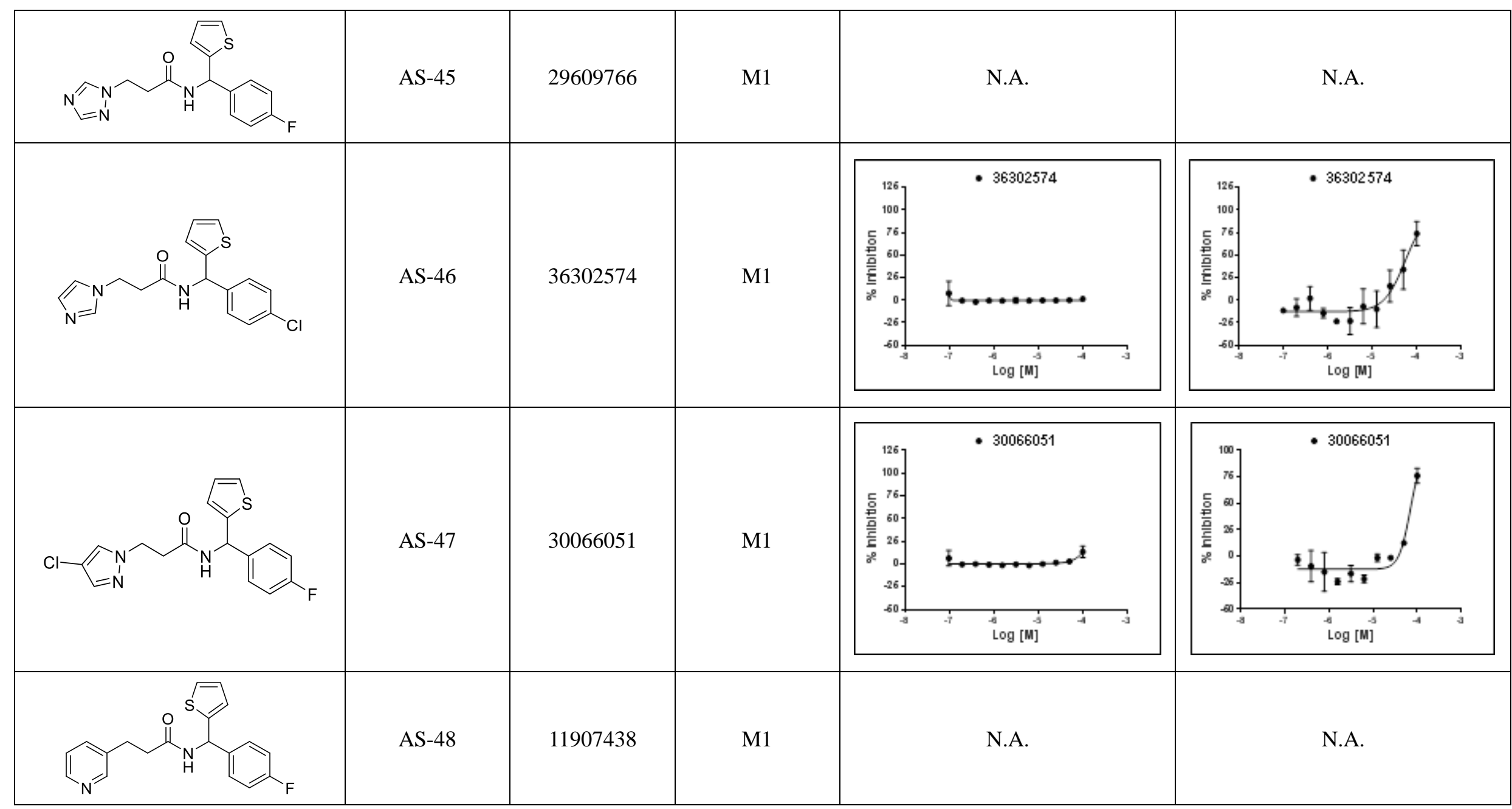




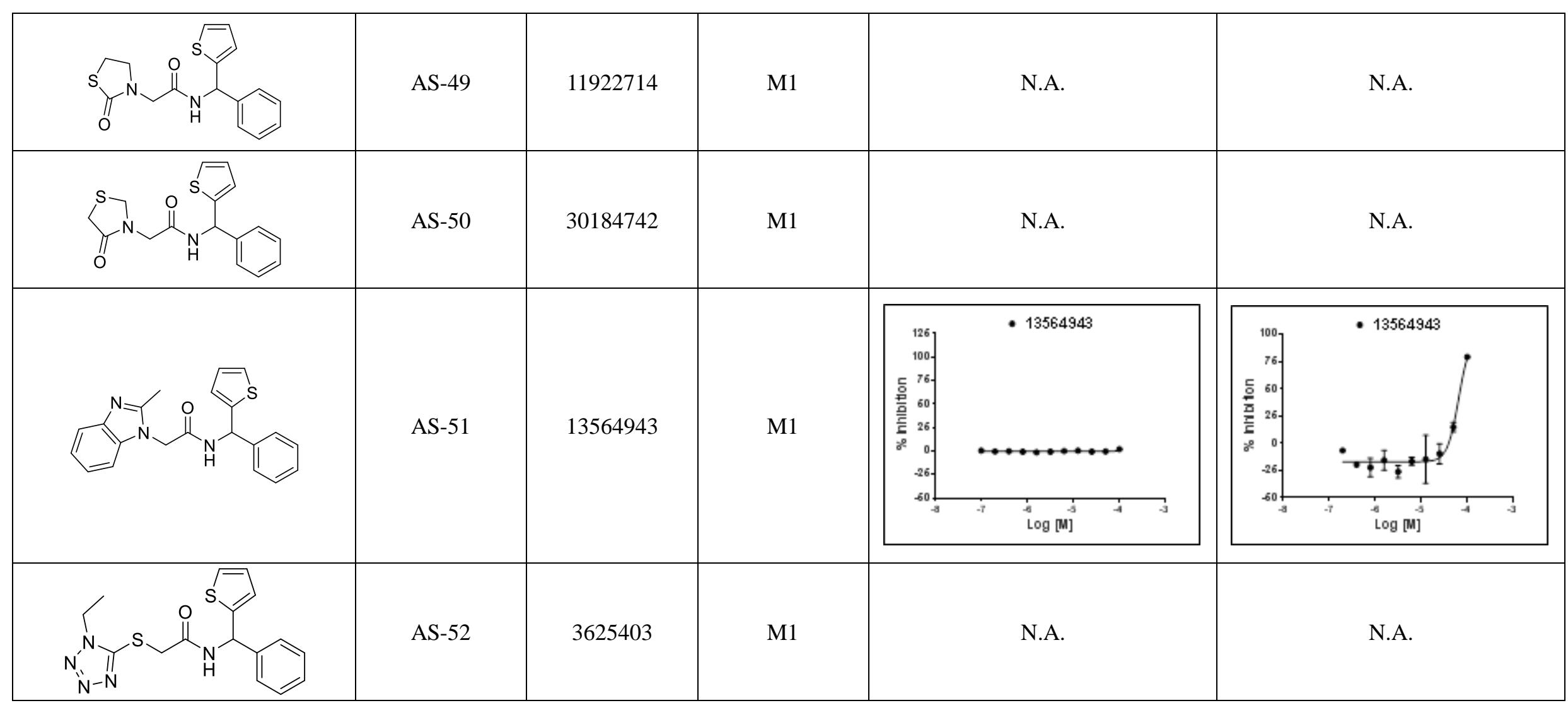




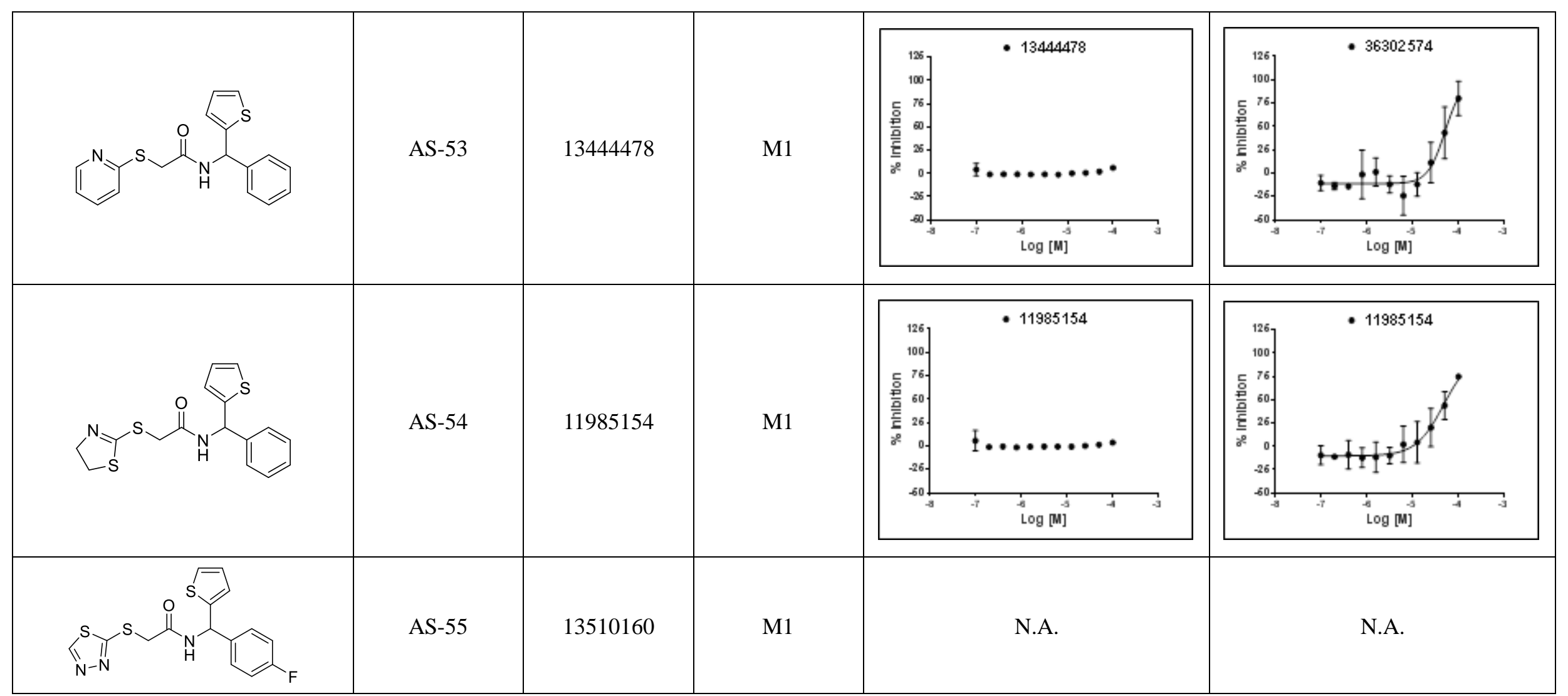




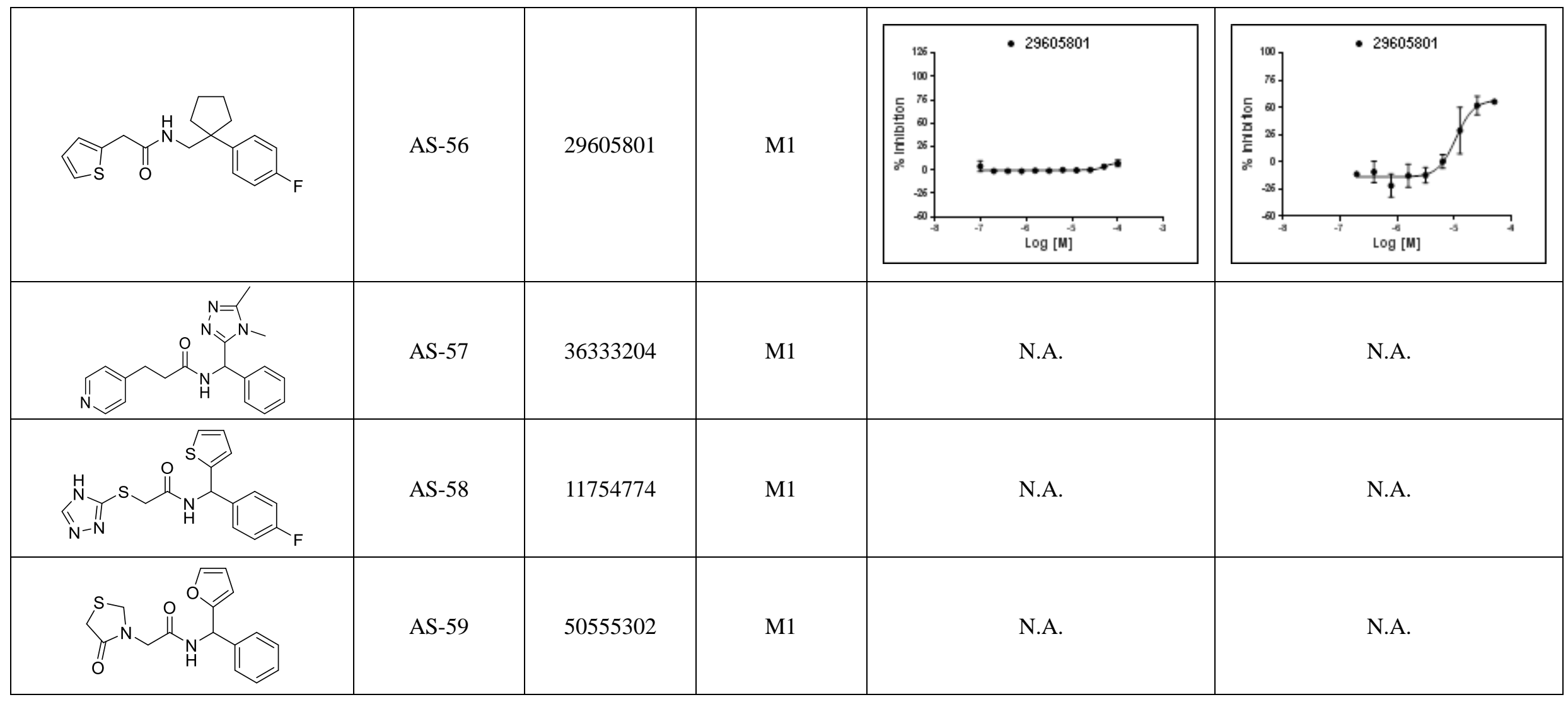

27 


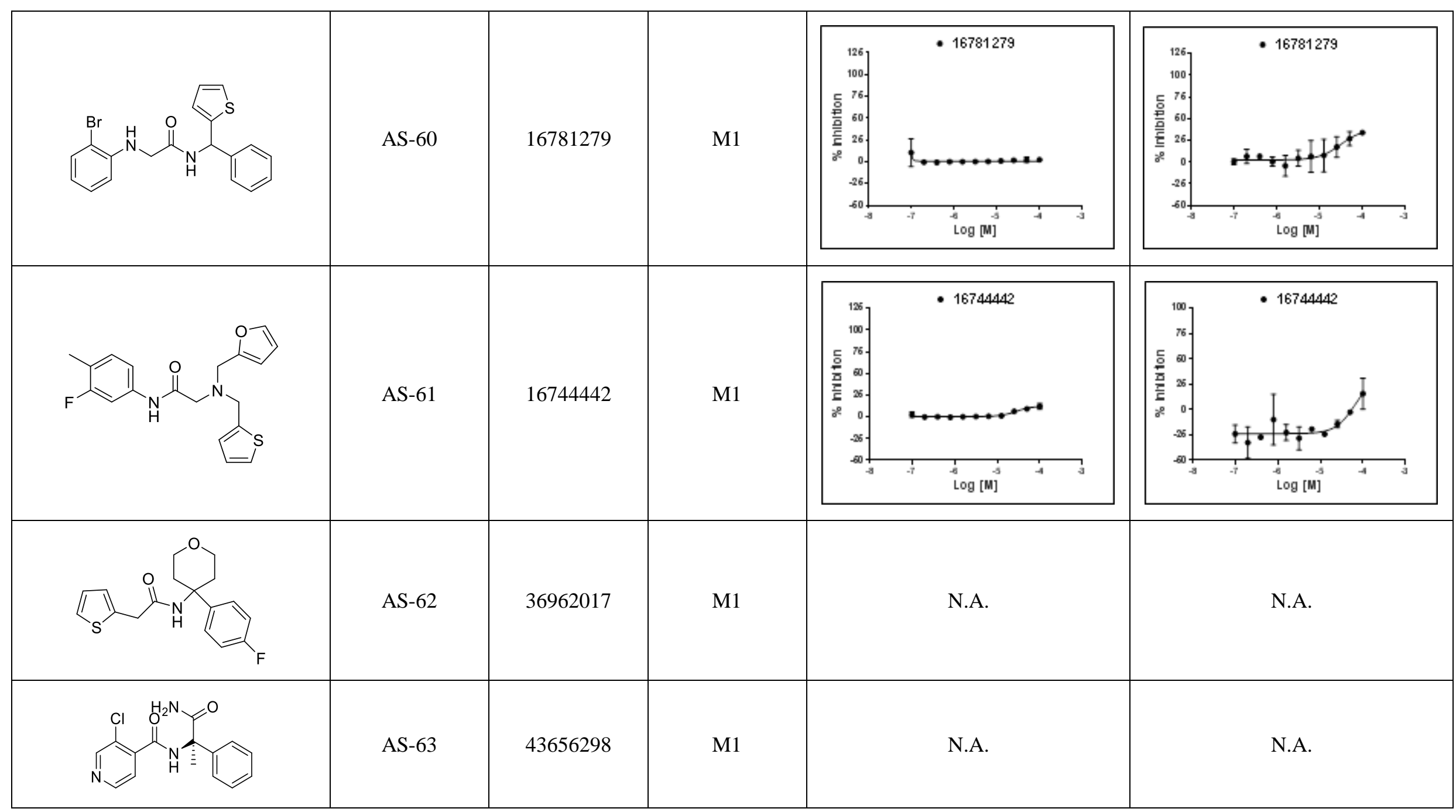

N.A.: no activity 
Table S4. Summary of the detected CIPs in the candidate compounds for $\beta$-AR

\begin{tabular}{|c|c|c|}
\hline Candidate Compound & CIP1 & CIP2 \\
\hline AS- $01 *$ & $\sqrt{ }$ & $\sqrt{ }$ \\
\hline AS-02* & $\sqrt{ }$ & $\sqrt{ }$ \\
\hline AS-03* & $\sqrt{ }$ & $\sqrt{ }$ \\
\hline AS-04* & & $\sqrt{ }$ \\
\hline AS-05* & $\sqrt{ }$ & $\sqrt{ }$ \\
\hline AS-06* & $\sqrt{ }$ & $\sqrt{ }$ \\
\hline AS-07 & $\sqrt{ }$ & \\
\hline AS-08 & $\sqrt{ }$ & \\
\hline AS-09* & $\sqrt{ }$ & $\sqrt{ }$ \\
\hline AS-10 & & $\sqrt{ }$ \\
\hline AS-11* & $\sqrt{ }$ & \\
\hline AS-12* & $\sqrt{ }$ & $\sqrt{ }$ \\
\hline AS-13* & $\sqrt{ }$ & \\
\hline AS-14 & $\sqrt{ }$ & $\sqrt{ }$ \\
\hline AS-15 & $\sqrt{ }$ & $\sqrt{ }$ \\
\hline AS-16 & & $\sqrt{ }$ \\
\hline AS-17* & $\sqrt{ }$ & $\sqrt{ }$ \\
\hline AS-18* & $\sqrt{ }$ & $\sqrt{ }$ \\
\hline AS-19 & $\sqrt{ }$ & $\sqrt{ }$ \\
\hline AS-33 & & $\sqrt{ }$ \\
\hline AS-34* & $\sqrt{ }$ & $\sqrt{ }$ \\
\hline AS-35 & $\sqrt{ }$ & $\sqrt{ }$ \\
\hline AS-36* & $\sqrt{ }$ & $\sqrt{ }$ \\
\hline AS-37* & $\sqrt{ }$ & $\sqrt{ }$ \\
\hline AS-38 & $\sqrt{ }$ & $\sqrt{ }$ \\
\hline AS-39 & $\sqrt{ }$ & \\
\hline AS-40 & $\sqrt{ }$ & $\sqrt{ }$ \\
\hline
\end{tabular}

* Active compounds for $\beta$-AR 
Table S5. Summary of the detected CIPs in the candidate compounds for mAChR

\begin{tabular}{|c|c|c|c|}
\hline Candidate Compound & CIP1 & CIP2 & CIP3 \\
\hline AS-20 & $\sqrt{ }$ & $\sqrt{ }$ & \\
\hline AS-21 & $\sqrt{ }$ & $\sqrt{ }$ & $\sqrt{ }$ \\
\hline AS-22 & $\sqrt{ }$ & $\sqrt{ }$ & \\
\hline AS-23 & $\sqrt{ }$ & $\sqrt{ }$ & \\
\hline \multicolumn{4}{|l|}{ AS-24 } \\
\hline AS-25 & $\sqrt{ }$ & $\sqrt{ }$ & \\
\hline AS-26 & $\sqrt{ }$ & $\sqrt{ }$ & \\
\hline AS-27 & $\sqrt{ }$ & $\sqrt{ }$ & \\
\hline AS-28 & $\sqrt{ }$ & $\sqrt{ }$ & $\sqrt{ }$ \\
\hline \multicolumn{4}{|l|}{ AS-29 } \\
\hline AS-30 & & $\sqrt{ }$ & \\
\hline AS-31 & $\sqrt{ }$ & $\sqrt{ }$ & \\
\hline AS-32 & & $\sqrt{ }$ & \\
\hline AS-41 & & & $\sqrt{ }$ \\
\hline AS-42 & $\sqrt{ }$ & $\sqrt{ }$ & \\
\hline AS-43 & & $\sqrt{ }$ & $\sqrt{ }$ \\
\hline AS-44 & & & $\sqrt{ }$ \\
\hline AS-45 & $\sqrt{ }$ & $\sqrt{ }$ & \\
\hline AS-46* & & $\sqrt{ }$ & \\
\hline AS-47* & & $\sqrt{ }$ & \\
\hline \multicolumn{4}{|l|}{ AS-48 } \\
\hline AS-49 & $\sqrt{ }$ & $\sqrt{ }$ & $\sqrt{ }$ \\
\hline AS-50 & $\sqrt{ }$ & $\sqrt{ }$ & $\sqrt{ }$ \\
\hline AS-51* & $\sqrt{ }$ & $\sqrt{ }$ & \\
\hline AS-52 & $\sqrt{ }$ & $\sqrt{ }$ & $\sqrt{ }$ \\
\hline AS-53* & $\sqrt{ }$ & $\sqrt{ }$ & \\
\hline \multicolumn{4}{|l|}{ AS-54* } \\
\hline \multicolumn{4}{|l|}{ AS-55 } \\
\hline AS-56* & $\sqrt{ }$ & $\sqrt{ }$ & \\
\hline AS-57 & $\sqrt{ }$ & & \\
\hline \multicolumn{4}{|l|}{ AS-58 } \\
\hline AS-59 & $\sqrt{ }$ & $\sqrt{ }$ & \\
\hline AS-60* & $\sqrt{ }$ & $\sqrt{ }$ & $\sqrt{ }$ \\
\hline AS-61* & & $\sqrt{ }$ & \\
\hline AS-62 & & $\sqrt{ }$ & \\
\hline AS-63 & & $\sqrt{ }$ & \\
\hline
\end{tabular}

* Active compounds for mAChR 